\title{
Structure and Circuit Solution of a Bidirectional Wireless Power Transmission System in Applied Robotics
}

\author{
Konstantin KRESTOVNIKOV, Ekaterina CHERSKIKH, Anton SAVELIEV
}

Laboratory of Autonomous Robotic Systems, St. Petersburg Federal Research Center of the Russian Academy of Sciences (SPC RAS), St. Petersburg Institute for Informatics and Automation of the Russian Academy of Sciences, 14th Line, VI, No. 39, 199178, St. Petersburg, Russia

k.krestovnikov@iias.spb.su, cherskikh.e@iias.spb.su, saveliev@iias.spb.su

Submitted September 1, 2020 / Accepted January 22, 2021

\begin{abstract}
In this paper the development of structure and circuit solution for a bidirectional wireless power transmission system is presented, based on current fed push-pull inverter. Existing solutions are analyzed, as well their structures and characteristics. The principle of operation of the developed circuit solution is described in receiving/ transmitting mode, an electrical principal circuit is given with respective ratios calculated. The SPICE modeling of the system was performed, and the theoretically calculated dependency of energy transmission efficiency from the transmitted power was obtained. The developed structure includes a step-up DC-DC converter, which enables to obtain the output voltage of the system, active in receiving mode, being equal or higher than the voltage of the power supply of energy-transmitting system. Therefore, the proposed bidirectional wireless power transmitting system can be utilized within a swarm robotic system with unified robots, having identical power supply batteries. Practical application of the proposed solution is relevant for energy transmission among autonomous robots, energy transmission from power supply source to robot and in reverse direction.
\end{abstract}

\section{Keywords}

Bidirectional wireless power transmission system, current fed push-pull inverter, swarm robotics, synchronous rectifier

\section{Introduction}

In the course of operation, autonomous robotic devices need routinely replenish their energy resource. In this case energy resource replenishment consists in battery recharge procedure, what is, essentially, energy transmission from the power source to the robot. Among the common solutions of this problem various wired and contacttype energy transmission approaches are particularly important. Both kinds of approaches assume presence of connection sockets or contact pairs and often require operator intervention into the robotic system operation process, what imposes certain constraints on the workflow. To reduce human involvement into the robot operation workflow and simplify the battery positioning and subsequent recharge process, wireless power transmission (WPT) devices may be employed. The most common solutions in this domain are based on electromagnetic induction and enable one-way energy transmission from the source to the consuming device. Unidirectional energy transmission, pertinent to these methods, limits their usage and precludes energy transmission among robotic devices such as [1] and [2], although such transmission mode is relevant in the domain of swarm robotics. Bidirectional energy transmission allows to go with a single wireless energy transmission system per robot to recharge the battery from power source and to transmit energy among robots.

Design and circuitry of bidirectional WPT systems is more complicated than in unidirectional ones and requires more complex and reliable control methods. Hence, there exists an obvious need of bidirectional WPT development, which would improve operational autonomy of robotic complexes and reduce human involvement into their operation.

Mathematical model of bidirectional WPT control, enabling energy transmission from one transmitter to several receivers at once, and its operability check using a prototype WPT system were described in [3]. Resonant circuit of every part of the system was built according to series-parallel design. System circuits are adjusted to work on a resonant frequency, and every rectifier is operated either in inverter mode or in rectifier mode, depending on the direction of energy transmission. The direction and value of the transmitted energy is defined by the opening angle of the controlled rectifier switches. In [3], a mathematical model is presented, which demonstrates the following: value and direction of the energy flow between several systems may be controlled using phase and amplitude modulation of voltage, where the energy flows can be used together or separately. WPT system, presented in this paper, has the following characteristics: power $1500 \mathrm{~W}$, operation frequency $20 \mathrm{kHz}$, energy transmission system efficiency $85 \%$ by air gap $40 \mathrm{~mm}$, supply voltage $200 \mathrm{~V}$. 
In [4], a hybrid bidirectional WPT system is proposed. The system consists of two pairs of coils. Here one pair acts as a transmitting part, and another one as a receiving part. Both the transmitting part of the system and the receiving one have two circuits, connected at common leg, whereas one of the circuits is designed according to serial topology and another one according to serial-parallel topology. Phase modulation is utilized to regulate the amplitude of the voltage, generated in the receiving circuit. The maximum transmitted power of the system is $3.3 \mathrm{~kW}$ with energy transmission efficiency of $91 \%$ by optimal layout of transmitting and receiving circuits relative to each other.

For faster battery recharge, which also could retain the battery service life, a bidirectional WPT system for electric cars is proposed in [5]. This energy transmission system has power of $6.6 \mathrm{~kW}$ and a constant operational frequency of $20.3 \mathrm{kHz}$ irrespective from the air gap. Supply voltage of the transmitting part is $400 \mathrm{~V}$, whereas the output voltage of the receiving part varies from 350 to $450 \mathrm{~V}$, maximum current in the coil is 16.5 A. Energy transmission efficiency under maximum load depending on the air gap size equals from 88.1 to $95.3 \%$. Sustainable system performance with coil diameter of $500 \mathrm{~mm}$ is achieved by air gap size under $200 \mathrm{~mm}$.

To increase the energy transmission efficiency in low power conditions, frequency control and hybrid frequencyphase control were proposed in [6]. Using hybrid control, it is convenient to maintain the operational frequency closer to the resonance frequency within the whole frequency setting range, what is more difficult within the usual frequency control approach. The proposed hybrid control strategy enables transmitted power setting in the broad range from 0 to $1.8 \mathrm{~kW}$ in a limited frequency range of $154 \mathrm{kHz}$. The proposed invertor with hybrid control uses phase shift to control power level. Maximum output power of the energy transmission system is up to $1.8 \mathrm{~kW}$, nominal transmitted power is $1 \mathrm{~kW}$ by air gap of $200 \mathrm{~mm}$ and voltage of $100 \mathrm{~V}$. In [7], a bidirectional WPT system for electric cars is proposed, with relatively high intended capacity: $22 \mathrm{~kW}$. To exclude high reactive currents, a serial resonance circuit is employed. The prototype system has the following characteristics: coil dimensions $600 \times 600 \mathrm{~mm}$, nominal transmitted power $11 \mathrm{~kW}$. Energy transmission distance remained unclear since it was not specified by the authors. This WPT system is powered from a DC source with voltage of $300 \mathrm{~V}$ and useful current of $35 \mathrm{~A}$. The energy transmission efficiency reaches $96 \%$ in this device. The system consists of two parts: mobile and stationary ones. Alteration of transmitted power value during operation on resonance frequency is enabled via adjustment of the operational frequency via pulse-width modulation on the lower control frequency. The converter can work based on zero switching of power switches in the whole range of the transmitted power. Using pulse-width modulation for transmitted power adjustment, a higher system efficiency can be ensured, than the pulse-frequency modulation. When the transmitted power of the system is $11 \mathrm{~kW}$, both methods ensure a virtually equal efficiency.
When a parallel resonance circuit is used in the transmitting part of WPT system, high voltage ripples arise on inverter switches, and, to compensate for them, in [8] it is proposed to add a capacitor to the system, which should be mounted serially with the resonance circuit. This circuit solution enables establishment of a serial-parallel resonance circuit in the system. Simultaneous application of resonance circuits of such type in both parts of the system (transmitting and receiving ones) causes system instability. Therefore, in this paper the serially-parallel resonance circuit is applied only in the transmitting part of the system, whereas in the receiving part a serial resonance circuit is used. For experimental testing of circuit solutions, a prototype system was assembled with power of $1.2 \mathrm{~kW}$, consuming input voltage of $325 \mathrm{~V}$ and taking input current of 3.7 A. Power switching frequency corresponds to the resonance performance mode of $50 \mathrm{kHz}$. The resulting efficiency of the system approaches to $92 \%$.

Authors of [9] presented a methodology for achieving the optimal design of a bidirectional WPT, where the circuits of the transmitting and receiving parts are assembled in serial-parallel way. The $8-\mathrm{kW}$ prototype works at a resonance frequency of $40 \mathrm{kHz}$ with efficiency of $92 \%$.

Authors of [10] develop an integrated circuit, which includes a bidirectional WPT control system, and implements a battery-to-battery energy transmission system on it. Every coil has four windings with inner diameter of $19 \mathrm{~mm}$ and outer diameter of $33 \mathrm{~mm}$. Air gap between the receiving and the transmitting part of the system is $6 \mathrm{~mm}$. The power supply voltage in the transmitting part is $4.2 \mathrm{~V}$, current amplitude in the coil is about $0.8 \mathrm{~A}$. Current amplitude in the coil of the receiving part is equal to $0.7 \mathrm{~A}$, output voltage is $4.1 \mathrm{~V}$. This wireless energy transmission system is operated at frequency of $6.78 \mathrm{MHz}$, whereas its output power is $1.55 \mathrm{~W}$. In nominal operating mode the system shows efficiency of $58.6 \%$.

Axial shifts and changes of air gap size between receiver and transmitter in wireless energy transmission systems, operating at resonance frequency, cause the decrease of energy transmission efficiency. To compensate for shift influence on transmitted power, authors of [11] propose a system with adjustable resonance frequency. This solution is implemented to account for the power losses, arising by air gap size change. The resonance circuit is controlled by frequency modulation. Voltage amplitude at the transmitting and receiving coils is $13 \mathrm{~V}$, this voltage is converted via synchronous buck-boost mechanism from the input and output voltage of $4 \mathrm{~V}$. The operating frequency of the system is adjusted in the range from $110 \mathrm{kHz}$ to $205 \mathrm{kHz}$. The round-shaped coil with inner diameter of $21.7 \mathrm{~mm}$ and outer diameter of $32 \mathrm{~mm}$ has 18 windings. The input and output voltage of the energy transmission system is 4 volts, transmitted power is $2.5 \mathrm{~W}$, voltage amplitude at coils of receiving and transmitting circuits is $13 \mathrm{~V}$, current amplitude at coils is $0.625 \mathrm{~A}$. With air gap size of $2 \mathrm{~mm}$ and lateral shifts up to $8 \mathrm{~mm}$ energy transmission efficiency is $70 \%$. 
Authors of [12] propose a controller for bidirectional power flow adjustment without information transfer to control the system between the receiving and the transmitting part. The proposed controller can be utilized as with unidirectional, as with bidirectional WPT, handling one or several workloads at a time. The value of the transmitted energy and direction of its transfer is controlled via phase shift of control signals with key opening. If the value of the power switch opening phase equals to 180 degrees, no energy transmission in the system occurs, and the values of 0 and 360 degrees correspond to the maximum transmitted energy in forward and backward directions, respectively. For operability check of the proposed controller, a prototype system was assembled with power of $1.5 \mathrm{~kW}$, consisting of a receiving part, transmitting part, and a control system. This system of bidirectional energy transfer has operating frequency of $20 \mathrm{kHz}$ and shows energy transmission efficiency of $85 \%$ by a $55-\mathrm{mm}$ wide air gap.

In the paper [13], a bidirectional system for transmission of energy and information is presented. The transmitted power is adjusted using phase shift of the power switch opening angle. The operational frequency of the system is $500 \mathrm{kHz}$. Robust operation of the system is ensured by the air gap up to $50 \mathrm{~mm}$ and by lateral shift of the coils up to $20 \mathrm{~mm}$. The transmitted signal has an 8-bit encoding.

In the most of the wireless energy transmission systems considered here, serial and serial-parallel resonance circuit topologies are used, because axial coil shift, air gap size and load value change less seriously influence the energy transmission efficiency. WPT power increase positively affects the efficiency and operation stability of the whole system by greater size of the air gap.

The analysis performed here showed, that the majority of the WPT systems belongs to the high-power class (above $1 \mathrm{~kW}$ ) and have great outer dimensions, as they are intended for use in electric cars. The second-large category is comprised of low-power systems $(1-10 \mathrm{~W})$ to be used in mobile autonomous electronics, these devices are much more compact, than the systems for the electric vehicles. Prototypes of the systems considered here are assembled on the basis of the half-bridge of bridge inverters. They require a dedicated control system, which implements bidirectional energy transmission algorithms. One of the common downsides of the most systems considered here is the need of operational frequency adjustment, depending on the mutual positioning of the receiving and the transmitting parts and workload value. When the system goes out of the resonance, the overall power and efficiency of the system decreases. The systems, employed in electric transport and mobile electronics, require operator intervention into mutual positioning of the receiving and transmitting parts of the system. Such intervention further ensures its performance in resonance mode. The purpose of this paper is development of a moderate-power bidirectional WPT system (operational range from $10 \mathrm{~W}$ to $1 \mathrm{~kW}$ ), which will work in resonance mode with high efficiency and transmitted power, irrespective of the mutual positioning of the receiving and the transmitting parts of the system.

\section{Design of the Bidirectional WPT System}

In earlier works we developed some unidirectional WPTs, equipped with cored and coreless coils [14], [15]. Within the earlier research we revealed, that developed prototypes with coreless coils are characterized by minor weight, better efficiency, and greater energy transmission distance, compared to the similar devices. Unidirectional energy transmission systems are easily compatible with various mobile service platforms [16]. Similar research, concerning energy resource distribution in robotic swarm [17], justified the need of development of bidirectional systems. The findings, made in context of the unidirectional systems, enabled the development of bidirectional WPT transmission system, presented in Fig. 1.

The main system unit is the resonant self-oscillator, that can be operated as a synchronous rectifier. Energy transmission is performed using electromagnetic induction via inductively-coupled parallel resonance circuits. The resonance circuits, in turn, are frequency-setting (1) for the self-oscillator, if the system operates in power transmitting mode:

$$
f=\frac{1}{2 \pi \sqrt{L C}}
$$

where $L$ is coil inductance, $\mathrm{H}$, and $C$ is capacity, F.

Resonant circuits are identical, and the system is generally symmetrical relative to inductively-coupled circuits. DC-DC boost converter is used here, hence EMF, induced in the receiving coil, has a lesser amplitude, compared to the EMF from the transmitting coil, because of the active losses in the resonance circuits, as well because of the air gap with high magnetic resistance. EMF amplitude in the transmitting coil is calculated according to $(2)[18,19,20]$ :

$$
U_{\mathrm{m}}=\pi V_{\mathrm{dc}}
$$

where $V_{\mathrm{dc}}$ is source voltage.

Therefore, with boost converter involved, it is possible to obtain output voltage equal to source voltage, when the system operates in receiver mode, and above source level, when the system operates in transmitter mode. Because of this feature, the system can be used for energy redistribution among autonomous devices, where the energy is supplied from batteries with equal operational voltage.

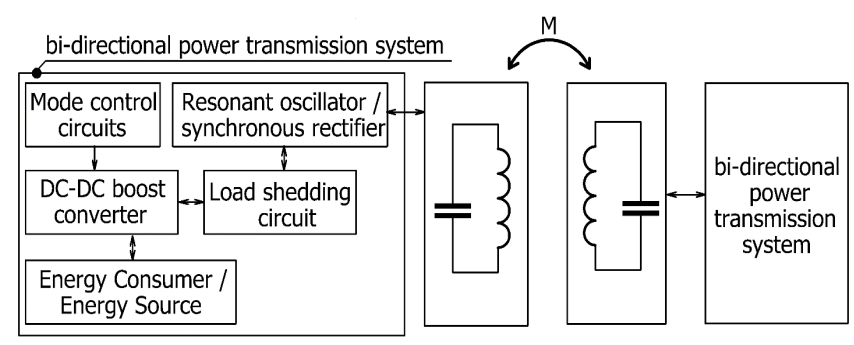

Fig. 1. Flowchart of the bidirectional WPT system. 


\section{Circuit Solution of the Bidirectional WPT system}

Circuit solution of the bidirectional WPT system, presented in Fig. 2 and 3, is developed based on previous research. Within those research papers WPT system on coreless coils were described [14], [21] and a synchronous rectifier was developed, as well synchronous rectifier losses were calculated to use such rectifier in the receiving part of WPT system [22], [23].

The receiving and the transmitting parts of WPT system are based on identical circuit solutions. In Fig. 2, the first part of circuit including a self-oscillator is shown, which can be operated in the synchronous rectifier mode and ensures smooth load connection.

The self-oscillator is implemented on transistors VT1 and VT2, and the transmitting/receiving resonance circuit L1C1 works as its frequency-setting circuit. The principle of circuit operation, which ensures smooth load connection, consists in the following: we increase the timeframe, while the transistor VT3 remains open, whereas the overall process duration is defined by the values C3 and R6, as well by VT3 parameters.

The second part of the principal diagram is presented in Fig. 3. The parts of the principal diagram of the bidirectional energy transmission system are connected in the points labeled by «+SR/RO» and «GND». The second part

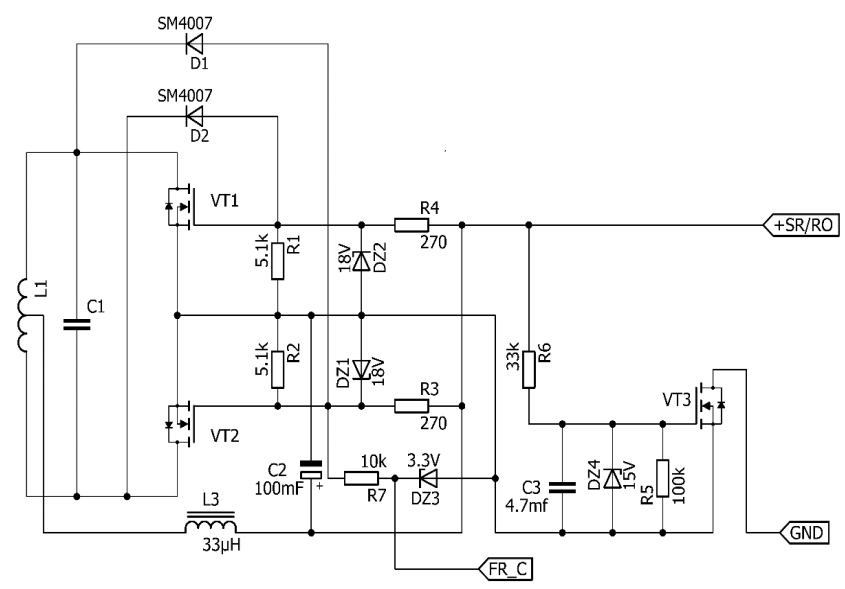

Fig. 2. Principal diagram of bidirectional WPT system, part 1 .

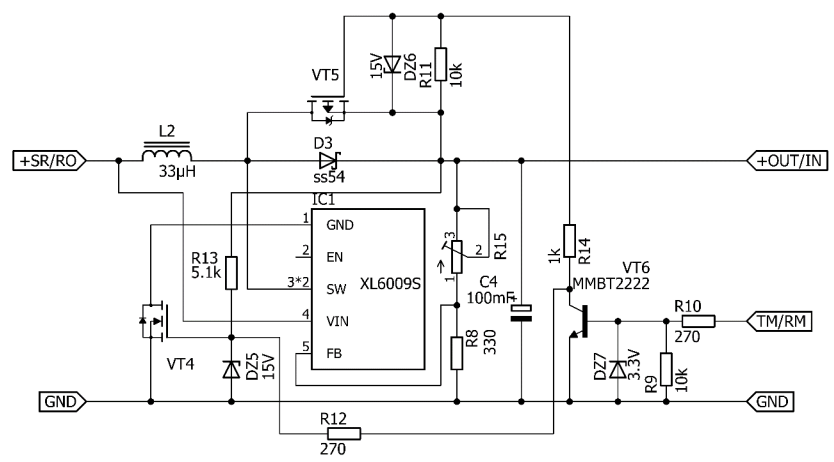

Fig. 3. Principal diagram of bidirectional WPT system, part 2. of the principal diagram includes a DC-DC boost converter, based on XL6009 chip, as well switching circuits on transistors VT4 and VT5.

\section{WPT System Operating Principle in Energy Transmission Mode}

Switching of WPT system into energy transmission mode is triggered by a high-level signal, transmitted through the circuit, labeled as «TM/RM», while the power supply is connected to the circuit between the points «+OUT/IN» and «GND». Before that, it should be ensured, that no rectangular signal exists in the frequency control point, labeled as «FR_C», where the label indicates the operating mode of the system. If a rectangular signal with operating frequency of the system is active, it indicates, that the bidirectional WPT operates in receiver mode, and switching the system into energy transmission mode is not permitted. High-level signal in the «TM/RM» circuit is fed via limiting resistor R10 to the base of the VT6 transistor and opens it. VT6 activation causes VT4 to close and VT5 to activate itself. VT4 closure disables the internal circuits of DC-DC converter, and VT5 bypasses the D3 diode; therefore, the «+» of power supply is connected to the point «+SR/RO». After the supply voltage has been enabled between the points «+SR/RO» and «GND», the current begins to flow to the VT3 gate, opening it and therefore bypassing its internal diode. As well, this current goes to the gates of the field MOSFET-transistors VT1 and VT2, charging the gate capacities via R3 and R4 resistors, thereby triggering the self-oscillator. To ensure reliable transistors activation and decrease of dynamic losses, it was empirically revealed, that calculation of the required value of the gate resistors $R_{3,4}$ can be performed according to (3):

$$
R_{3,4}=\frac{1}{30 C_{\mathrm{g}} f}
$$

where $C_{\mathrm{g}}$ is the gate capacity of the transistors in action. Equation (3) is derived on premise, that the gate capacity charging should occur within a $1 / 10$ period.

Calculation methodology, presented in [22], can be used for loss calculation in the self-oscillator, which operates in energy transmission mode, whereas loss calculation in the resonance circuit may be performed as follows (4):

$$
P_{\mathrm{LC}}=\sqrt{2} I_{\mathrm{m}}^{2}\left(R_{\mathrm{l}}+E_{\mathrm{sr}}\right)
$$

where $R_{1}$ is active resistance of loop coil, and $E_{\mathrm{sr}}$ is equivalent serial resistance of loop capacitor.

\section{WPT System Operating Principle in Energy Reception Mode}

The operation of WPT system switching into energy reception mode is triggered by a low-level signal, trans- 
mitted into the circuit. This circuit is labeled as «TM/RM». In this case the transistor VT5 is deactivated, and its internal diode is connected parallel to D3. Further VT4 will be activated, as the required voltage level will be reached at point «+SR/RO», and the current will flow to its gate through the throttle of the DC-DC boost converter and D3 diode. After start of the bidirectional WPT system in energy reception mode, EMF is induced in the coil L1, which acts as the receiving one in this operating mode, and the electrolytic capacity $\mathrm{C} 2$ begins to charge via internal diodes of the MOSFET transistors. As soon $\mathrm{C} 1$ accumulates the baseline voltage, needed to activation the MOSFET channel (VT1, VT2), the transistor opens, and bypasses the internal diode through an open channel, having much lower resistance, than the dynamic resistance of diode. Diodes D1, D2 should prevent through current on transistors VT1, VT2. The D1 diode is blanking the transistor VT2 on the opposite rectifier leg, while the transistor VT1 is open. This occurs due to the fact that the cathode of the diode D1 is connected to the ground while VT1 is open, and it discharges the gate of transistor VT2. The latter pair on the other leg (diode D2 and transistor VT1) performs equally to the former one (diode D1 and transistor VT2). R1, R2 enable the gate to discharge and the transistor to close, when the receiving coil is devoid of EMF. The DZ1-R4 and DZ2-R3 circuits make up a parametric stabilizer, needed to limit the voltage in the gate-source transistor circuit. Also, R3, R4 resistors enable charging of gate element, when the transistor opens. $\mathrm{C} 1$ and L2 comprise the smoothing rectifier filter; here the $\mathrm{C} 1$ element is also necessary to store power, needed to supply the power switches. The voltage on $\mathrm{C} 2$ capacitor, while the system operates in energy reception mode, is calculated according to (5):

$$
U_{\mathrm{m}}=k_{\mathrm{c}} \frac{\pi V_{\mathrm{dc}}}{\sqrt{2}}
$$

where $k_{\mathrm{c}}$ is coupling coefficient between the inductively coupled receiving and transmitting coils.

When the rectified voltage arises in the point «+SR/RO», capacitor C3 begins to charge via resistor R6, as well as the gate capacity VT3 connected to it in parallel mode. Transistor VT3 opens smoothly, connecting the DCDC converter to the resonance self-oscillator, which operates in rectifier mode. Further transistor VT4 triggers, and DC-DC boost converter starts. Then at output of the reverse wireless energy transmission system at the point «+OUT/IN» output voltage arises, which value is set by the adjustable resistor R15.

\section{SPICE Modeling of the Developed Circuit}

Initial operability check of the proposed circuit solution was performed in a SPICE simulator. The original circuit solution was simplified for this model by omitting the DC-DC converter; though, in the calculation of the output power and efficiency, the efficiency of the DC-DC converter was taken to be $87 \%$. Transistors VT3 were re- placed by the active resistance of $0.065 \mathrm{Ohm}$ in the receiving and in the transmitting parts of the system. The transistor VT5 also was replaced by the active resistance of $0.065 \mathrm{Ohm}$ in the transmitting part of the system. Equivalent serial resistance of the capacitor of the resonant circuit and active resistance of the coil were taken equal to $0.01 \mathrm{Ohm}$. Here the inductance of the receiving and transmitting coil L1 equals to $14 \mu \mathrm{H}$, capacitance of the resonant capacitor $\mathrm{C} 1$ equals to $0.47 \mu \mathrm{F}$. Based on our previous research, the coupling coefficient of the coils of the receiving and the transmitting parts of the system was taken to be $k_{\mathrm{c}}=0.85$, this value could be achieved with the coils being in close proximity to each other. The curve of dependency of the energy transmission efficiency from the transmitted power, established upon the modeled data, is presented in Fig. 4.

Modeling of the circuit performance was performed for active load with resistance from 1 to $250 \mathrm{Ohm}$. The maximum output power of $8.81 \mathrm{~W}$ was achieved by load resistance of $2 \mathrm{Ohm}$. The maximum efficiency of $60.05 \%$ is achieved by output power of $3.73 \mathrm{~W}$. The current and voltage waveforms, reflecting the system performance without load are presented in Fig. 5, voltage of the power source of the transmitting system is $8.4 \mathrm{~V}$.

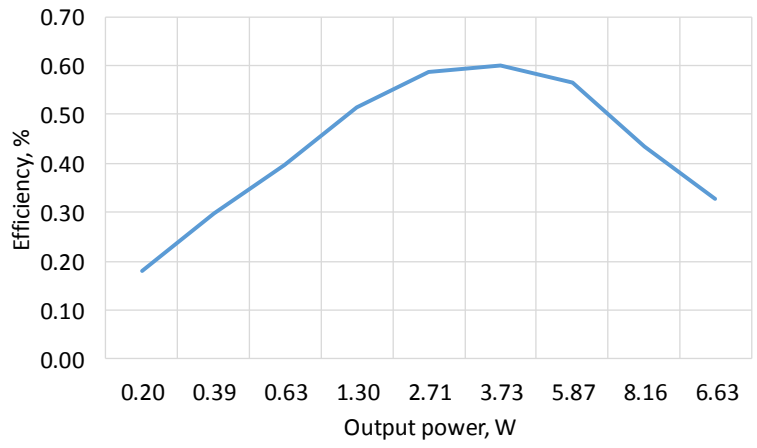

Fig. 4. Dependency of the energy transmission efficiency from the transmitted power.
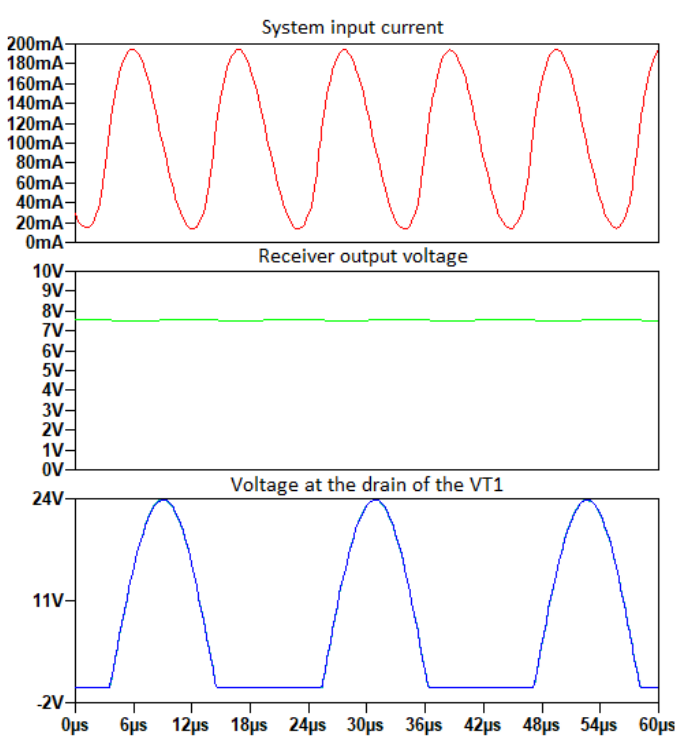

Fig. 5. Current and voltage waveforms by system performance without load. 
As follows from Fig. 5, the operational frequency of the system without load was $\sim 43 \mathrm{kHz}$, whereas the maximum level of output voltage of the receiving system was $7.56 \mathrm{~V}$.

The performed modeling enabled the experimental verification of the operability of the proposed circuit solution. It also enabled to derive the theoretical dependency of system efficiency from the transmitted power by specified parameters and constraints. For further stages of the research, experimental system prototypes were assembled.

\section{Experimental Verification of Circuit Design}

To test the developed circuit solution, experimental models of the system were assembled and mounted on mobile autonomous robotic platforms [24], and small replicas of these platforms, all having the same kinematic properties, were used for experiments. The system models, shown in Fig. 6, mounted on platforms, have identical electrical circuitry and resonant circuits.

Each of the coils of the resonant circuit has 12 turns with center tap, whereas the cross-section of the winding wire is $0.374 \mathrm{~mm}^{2}$. As resonant circuit capacitors, film MPP capacitors with a low value of equivalent series resistance were used here.

In the course of the experimental verification, system models were connected to Li-ion batteries of robotic platforms, as shown in Fig. 7, with an operating voltage of $7.4 \mathrm{~V}$ and capacity of $2500 \mathrm{~mA} / \mathrm{h}$.

One of the models was operated in energy transfer mode and the other model was operated in energy reception mode. Figure 8 shows the waveform of the voltage curves on the transmitting and receiving coils. Here the battery, connected to the system sample, operating in the energy reception mode is charged, and the output current has the minimum value. Consequently, the system operation without load.

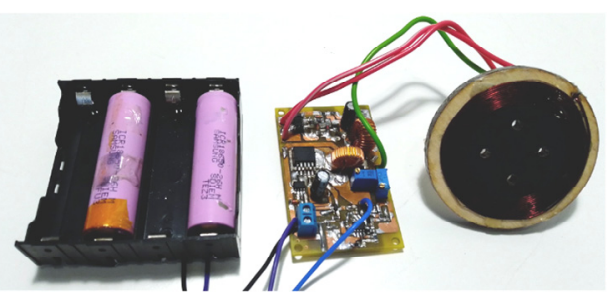

Fig. 6. Experimental model of the developed system.

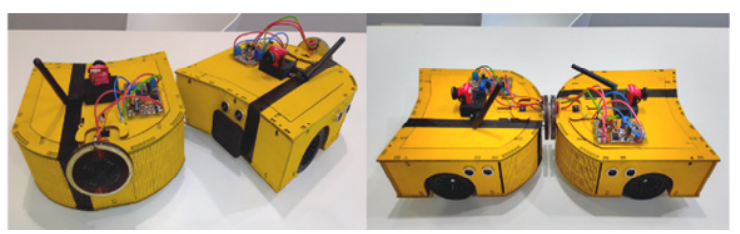

Fig. 7. Experimental models of the developed system mounted on mobile autonomous platforms.

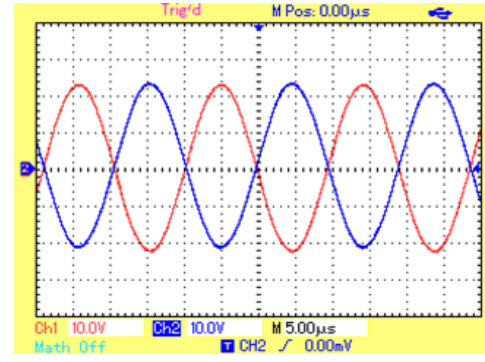

Fig. 8. The voltage curves on the transmitting and receiving coils with a charged battery.

In Fig. 8 and in subsequent figures, the voltage curves at the transmitting and receiving coil are indicated in red and blue respectively. The voltage values at the receiving and transmitting coils show sinusoidal shapes without distortion, and close amplitudes. The phase of the curves is shifted by 180 degrees, since the coils have the same winding path, and are mounted in the opposite direction to each other.

Figure 9 shows the voltage curves for a charging current of $0.38 \mathrm{~A}$, where distance between the coils differs. It is evident from Fig. 9 that, when the system is under load, the sinusoidal shape of the curves is preserved, but with some distortions. As the distance between the coils increases to $20 \mathrm{~mm}$, a change in the phase shift between the voltage values on the coils is observed.

Using scale-up, we successfully revealed insignificant distortions in voltage curve shape on the transmitting coil, as presented in Fig. 10.

When opening the transistor power keys of the selfoscillator, the transition process occurs; it is periodic as such,

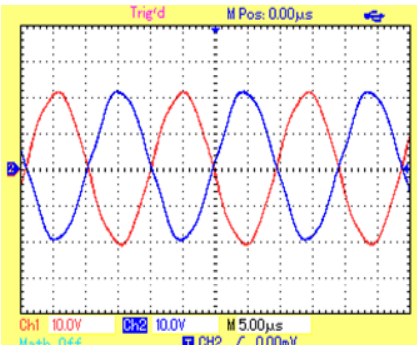

(a)

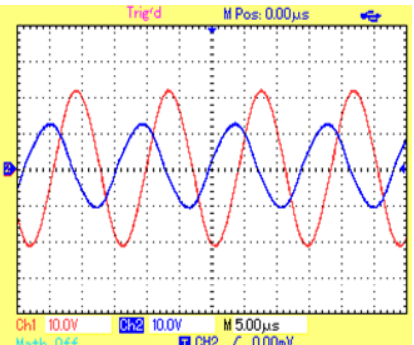

(b)
Fig. 9. The voltage curve shape on the transmitting and receiving coils during battery charging process. Two coils are in close proximity (a), distance of $20 \mathrm{~mm}$ is between the coils (b).

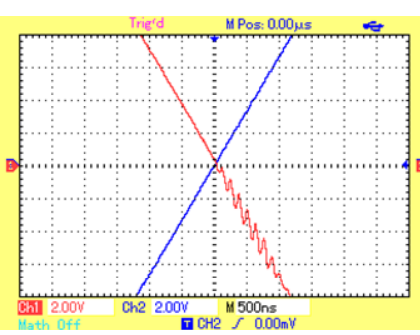

(a)

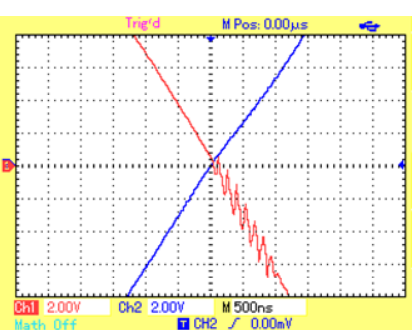

(b)
Fig. 10. Transition process when opening transistors of the oscillator. No load (a), at a load current of $0.38 \mathrm{~A}$ (b). 
and increase in the load current of the system causes the increase in the amplitude of the oscillations. The presence of this transient stage increases the losses on the transistors, which can adversely affect the overall efficiency of the system with a significant transmitted power. To reduce the amplitude and time of the transition process is possible by reducing the resistance value of the gate resistors R3 and R4.

\section{Discussion}

In contrast to other research papers in this domain, in our paper particular attention is paid to the circuitry and operating principle of the bidirectional energy transmission system. The majority of works considered in the context of this research, primarily deal with control systems, without detailed considerations of the system structure, circuitry and operating principles. The systems, proposed in the reviewed literature, typically have a power unit, implemented with bridge and half-bridge inverters with a dedicated control system, or as fully integrated solutions.

The advantages of the bidirectional WPT system, considered here, over the similar solutions from the other reviewed work, consist in implementation of an integrated control system, which can be operated in energy transmission mode and in the presence of a power unit, which does not require a dedicated control system, because it is implemented as a resonant self-oscillator. Using a resonant selfoscillator and identical circuits both in receiving and transmitting parts of the system, we ensure continuous performance in resonant mode, and such approach requires no operating frequency adjustment, irrespective of the distance between the coils and their shifts, what allows to boost the parameters of efficiency and transmitted power of the system. This feature allows to use the system by low alignment accuracy of the transmitting and receiving coils, thereby reducing resulting dimensions and weight of the whole system, preserving the required level of transmitted power, what is particularly relevant in autonomous robotics.

\section{Conclusions}

This paper presents structure and principal circuit diagram of a bidirectional WPT system, based on a self-oscillator with a parallel resonance circuit. Operating principle of the developed circuit solution is described in energy transmission and reception modes. SPICE-modeling and experimental verification of the operability of the proposed system in the modes of receiving and transmitting energy were performed, and waveforms of the voltage at the receiving and transmitting coils were obtained. The voltage curve at the receiving and transmitting coils has a sinusoidal shape with insignificant distortions when the system is working under load. The distinctive feature of the presented WPT system is that it enables to obtain voltage, equal to source voltage (in receiver mode) or higher than source voltage (in transmitter mode). The system, presented in this paper, can be utilized for redistribution of energy resources among the autonomous agents of robotic systems, powered by batteries of equal or different operating voltage. Further research will be aimed to derive performance characteristics of the developed circuit solution, as well to establish a calculation and optimization methodology to be used with receiving and transmitting coils.

\section{Acknowledgement}

This research is supported by the RSF Project No. 20-79-10325.

\section{References}

[1] NAGY, I. Behaviour study of a multi-agent mobile robot system during potential field building. Acta Polytechnica Hungarica, 2009, vol. 6, no. 4, p. 111-136. ISSN 1785-8860

[2] NAGY, I. From exploring to optimal path planning: Considering error of navigation in multi-agent mobile robot domain. Acta Polytechnica Hungarica, 2014, vol. 11, no. 6, p. 39-55. DOI: 10.12700/APH.11.06.2014.06.3

[3] MADAWALA, U. K., THRIMAWITHANA, D. J. A bidirectional inductive power interface for electric vehicles in V2G systems. Transactions on Industrial Electronics, 2011, vol. 58, no. 10, p. 4789-4796. DOI: 10.1109/TIE.2011.2114312

[4] ZHAO, L., THRIMAWITHANA, D. J., MADAWALA, U. K. Hybrid bidirectional wireless EV charging system tolerant to pad misalignment. Transactions on Industrial Electronics, 2017, vol. 64 , no. 9 , p. 7079-7086. DOI: 10.1109/TIE.2017.2686301

[5] LEE, J. Y., HAN, B. M. A bidirectional wireless power transfer EV charger using self-resonant PWM. Transactions on Power Electronics, 2014, vol. 30, no. 4, p. 1784-1787. DOI: 10.1109/TPEL.2014.2346255

[6] BOJARSKI, M., KUTTY, K. K., CZARKOWSKI, D., et al. Multiphase resonant inverters for bidirectional wireless power transfer. In Proceedings of International Electric Vehicle Conference (IEVC). Florence (Italy), 2014, p. 1-7. DOI: 10.1109/IEVC.2014.7056191

[7] TRITSCHLER, J., REICHERT, S., GOELDI, B. A practical investigation of a high power, bidirectional charging system for electric vehicles. In Proceedings of 16th European Conference on Power Electronics and Applications. Lappeenranta (Finland), 2014, p. 1-7. DOI: 10.1109/EPE.2014.6910809

[8] SAMANTA, S., RATHORE, A. K., THRIMAWITHANA, D. J. Bidirectional current-fed half-bridge (C)(LC)-(LC) configuration for inductive wireless power transfer system. Transactions on Industry Applications, 2017, vol. 53, no. 4, p. 4053-4062. DOI: 10.1109/TIA.2017.2682793

[9] MOHAMED, A. A., MARIM, A. A., MOHAMMED, O. A. Magnetic design considerations of bidirectional inductive wireless power transfer system for EV applications. IEEE Transactions on Magnetics, 2017, vol. 53, no. 6, p. 1-5. DOI: 10.1109/TMAG.2017.2656819

[10] HUANG, M., LU, Y., MARTINS, R. P. A reconfigurable bidirectional wireless power transceiver for battery-to-battery wireless charging. Transactions on Power Electronics, 2018, vol. 34, no. 8, p. 7745-7753. DOI: 10.1109/TPEL.2018.2881285

[11] MIURA, S., NISHIJIMA, K., NABESHIMA, T. Bi-directional wireless charging between portable devices. In Proceedings of 
International Conference on Renewable Energy Research and Applications (ICRERA). Madrid (Spain), 2013, p. 775-778. DOI: 10.1109/ICRERA.2013.6749857

[12] MADAWALA, U. K., NEATH, M., THRIMAWITHANA, D. J. A power-frequency controller for bidirectional inductive power transfer systems, IEEE Transactions on Industrial Electronics, 2011, vol. 60, no. 1, p. 310-317. DOI: 10.1109/TIE.2011.2174537

[13] WU, H., GU, B., WANG, X., et al. Design and control of a bidirectional wireless charging system using $\mathrm{GaN}$ devices. In Proceedings of Applied Power Electronics Conference and Exposition (APEC). Anaheim (CA, USA), 2019, p. 864-869. DOI: 10.1109/APEC.2019.8721909

[14] KRESTOVNIKOV, K., CHERSKIKH, E., SMIRNOV, P. Wireless power transmission system based on coreless coils for resource reallocation within robot group. In Proceedings of International Conference on Interactive Collaborative Robotics. Istanbul (Turkey), 2019, p. 193-203. DOI: 10.1007/978-3-03026118-4 19

[15] SAVELIEV, A., KRESTOVNIKOV, K., SOLENI, S. Development of a wireless charger for a mobile robot technical platform. In Proceedings of Intelligent Power Systems, Materials of the V International Youth Forum. Tomsk (Russia), 2017, p. 197-201.

[16] PAVLIUK, N., SMIRNOV, P., KOVALEV, A. Constructional and architectural solutions for service mobile platform with pluggable modules. Izvestiva Tula State University. Technical Science, 2019, vol. 10, p. 181-193. ISSN 2071-6168

[17] KRESTOVNIKOV, K., CHERSKIKH, E., RONZHIN, A. Mathematical model of a swarm robotic system with wireless bidirectional energy transfer A. Robotics: Industry 4.0 Issues \& New Intelligent Control Paradigms, Studies in Systems, Decision and Control, 2020, vol. 272, p. 13-23. DOI: 10.1007/978-3-030$37841-7$

[18] ABDOLKHANI, A., HU, A. P., TIAN, J. Autonomous polyphase current-fed push-pull resonant converter based on ring coupled oscillators. Journal of Emerging and Selected Topics in Power Electronics, 2015, vol. 3, no. 2, p. 568-576. DOI: 10.1109/JESTPE.2014.2377171

[19] ABDOLKHANI, A., HU, A. P. Improved autonomous current-fed push-pull resonant inverter. IET Power Electronics, 2014, vol. 7 , no. 8, p. 2103-2110. DOI: 10.1049/iet-pel.2013.0749

[20] HU, A. P., SI, P. A low cost portable car heater based on a novel current-fed push-pull inverter. In Proceedings Australasian Universities Power Engineering Conference (AUPEC 2004). Brisbane (Australia), 2004, p. 1-5
[21] Krestovnikov, K., CherskiKh, E., Shabanova, A. Circuit designs and engineering solutions based on synchronous rectifier for wireless energy transfer system. Modeling, Optimization and Information Technology, 2019, vol. 7, no. 4, p. 1-15. DOI: 10.26102/2310-6018/2019.27.4.018 (In Russian)

[22] KRESTOVNIKOV, K., CHERSKIKH, E., PAVLIUK, N. Concept of a synchronous rectifier for wireless power transfer system. In Proceedings of 18th International Conference on Smart Technologies (EUROCON 2019). Novi Sad (Serbia), 2019, p. 1-5. DOI: 10.1109/EUROCON.2019.8861856

[23] KRESTOVNIKOV, K., SAVELIEV, A., SHABANOVA, A., et al. Comparative study of synchronous and non-synchronous rectifiers for use in the receiving part of a wireless charging system. In Proceedings of 14th International Conference on Electromechanics and Robotics "Zavalishin's Readings". Tula (Russia), 2020, p. 675-685. DOI: 10.1007/978-981-13-9267-2_56

[24] PAVLIUK, N., KHARKOV, I., ZIMULDINOV, E., et al. Development of multipurpose mobile platform with a modular structure. In Proceedings of 14th International Conference on Electromechanics and Robotics "Zavalishin's Readings". Tula (Russia), 2020, p. 137-147. DOI: 10.1007/978-981-13-9267-2_12

\section{About the Authors ...}

Konstantin KRESTOVNIKOV was born in Orsk. He received his M.Sc. from Saint Petersburg State University of Aerospace Instrumentation in 2019. His research interests include wireless power transmission systems, mechatronics, and electronics.

Ekaterina CHERSKIKH (corresponding author) was born in Stary Oskol. She received her M.Sc. from Saint Petersburg State University of Aerospace Instrumentation in 2019. Her research interests include robotics, mechatronics, and electronics.

Anton SAVELIEV was born in Kazan. He received his Ph.D. from St. Petersburg Institute for Informatics and Automation of the Russian Academy of Sciences. His research interests include robotics, mechatronics, and programming. 